\title{
Fungal homoserine transacetylase: A potential antifungal target
}

\author{
Esra Seyran ${ }^{1}$ (1) \\ 'International Centre for Genetic Engineering and Biotechnology, Trieste, Italy
}

ORCID IDs of the authors: E.S. 0000-0002-0384-4300

Cite this article as: Seyran, E. (2021). Fungal homoserine transacetylase: A potential antifungal target. İstanbul Journal of Pharmacy, 51(1), 137-140.

\begin{abstract}
We are facing a significant challenge due to the tremendous rise in drug resistant fungal pathogen populations. The number of antifungal drugs is limited, as are the known targets they inhibit. The limited number of drugs and targets that have been successfully exploited in antifungal drug discovery is in part due to the eukaryotic nature of these organisms, which is shared with mammalian cells. An ideal antifungal substance is one that targets an indispensable pathway for pathogenic fungal survival that is not present in mammals or plants. Methionine is an essential amino acid for fungi and inhibition of its biosynthesis is deleterious for the pathogen. The biochemical pathways that produce methionine do not exist in mammals as they acquire it from their diet. Homoserine transacetylase, which catalyses the first committed step in methionine biosynthesis, therefore represents an attractive drug target. This enzyme is absent in mammals and unique to fungi and bacteria, suggesting that drugs targeting it would be of low toxicity, if any, to mammals, while having a lethal effect against the fungal pathogen. Homoserine transacetylase, based on its role in the production of methionine and its absence in mammals and plants could be a potential antifungal target.
\end{abstract}

Keywords: Antifungal, Homoserine Transacetylase, Methionine

\section{INTRODUCTION}

Fungal infections in general accounted for $40 \%$ of deaths due to hospital acquired infections. Several fungal pathogens, such as Coccidioides immitis, can infect healthy individuals, however, the majority of fungal infections are caused by opportunistic pathogens that attack immunosuppressed patients of HIV, chemotherapy or organ transplant (Pfaller \& Diekema, 2007). Developments in the treatments against HIV and cancer are leading to longer survival of immunocompromised individuals but, unfortunately, aggressive immunosuppressive therapy for organ transplant recipients and exposure to a broad spectrum antibiotics are also predisposing patients to fungal infections. Fungal infections in immunocompromised individuals are often life threatening. Mortality rates of hospitalized patients with candidiasis are approximately 50\% (Low \& Rotstein, 2011). Increased prevalence of Candida albicans clinical isolates resistant to traditional therapies calls for the need for novel agents containing higher specificity for fungal cells over mammalian cells (Ashman et al., 2004; Whaley et al., 2016). There is an urgent need for novel agents containing higher specificity for fungal cells over mammalian cells.

The novel fungal specific agents could potentially be identified by targeting unique biochemical activities that are present in $C$. albicans and other pathogenic fungi but which are absent in mammalian systems. The mechanism of action of conventional antifungal drugs can be broadly classified as those acting on fungal membrane permeability or on the enzymes of the ergosterol biosynthetic pathway. The selectivity of these antifungal agents is obtained by their ability to bind to sterols that are unique to fungal 
cells or by their ability to target fungus specific enzymes that are required for the production of the fungal cell membrane. The major class of antifungal agents in clinical use against candidiasis today are azoles. The azole class of antifungals act by inhibiting the biosynthesis of ergosterol and have a broad spectrum of fungistatic activity. Inhibition of cytochrome P450 dependent sterol 14a-demethylase by azoles leads to a depletion of ergosterol and an accumulation of 14a-methyl sterols, leading to disruption of the fungal cell membrane (Mazu, Bricker, Flores-Rozas, \& Ablordeppey, 2016). The azoles, fluconazole, itraconazole and ketoconazole are used frequently against invasive mucocutaneous candidiasis and cryptococcal meningitis (Chang, Yu, Heitman, Wellington, \& Chen, 2017).

Fungal resistance to azoles is currently a problem in both medicine and agriculture. Azole resistance initially appeared in barley powdery mildew isolates in the early 1980s, soon after azole resistance became prevalent in other important plant pathogens (Hollomon, 1993). Clinically, azole resistance is increasing in frequency, especially among HIV patients with C. albicans infections (Vandeputte et al., 2005). Resistance is often a consequence of treatment or prevention of mucocutaneous candidiasis with low doses of fluconazole over a long period of time. In the case of immunocompromised patients, infections often reoccur, and patients are often less responsive to azoles. Moreover, resistance to one azole drug can usually lead to cross-resistance to other azoles (Panackal et al., 2006). In C. albicans, azole resistance is mediated through more than one mechanism. Resistance can be the result of an alteration of the target enzyme, the cytochrome P-450 lanosterol 14a demethylase, either by overexpression or as a result of point mutations in the gene that encodes it. The former requires the need for a higher intracellular azole concentration to complex all the enzyme molecules present in the cells, and the latter leads to amino acid substitutions, resulting in a decreased affinity for azole derivatives. A second major mechanism is the failure of azole antifungal agents to accumulate inside the fungal cell as a consequence of enhanced drug efflux (Warrilow et al., 2019). The Ppolyene class of antifungals are macrolide antibiotics made up of alternating conjugated double bonds. The polyene drugs interact with ergosterol in fungal membranes and induce channels in the fungal membrane, resulting in the loss of membrane selective permeability. Cholesterol is a building block in human cell membranes with a similar in structure to ergosterol, and as a result there is some toxicity associated with the use of polyene class antifungals (Omura \& Tanaka, 1984). The other problem in hand is the high potential of current antifungal compounds for drug-drug interactions. For example, azoles are both a substrate and inhibitor of several cytochrome P450 enzymes, with CYP3A4 being the major enzyme involved. Other classes of antifungal drugs such as echinocandins may lead to pharmacodynamic interaction on the level of renal toxicity (Zager, 2000).

\section{Amino acid biosynthesis pathways as antifungal targets}

The development of new antifungal drugs with new and different mechanism of actions is critical in order to provide treatment to life threatening fungal diseases. The yeast genome study revealed that approximately 1,100 genes are required for fungal viability and around 350 of these genes have no homologues in the human genome (Ho et al., 2002). Amino acid biosynthesis has been considered as a potential target for antimicrobial drug development. Certain amino acids are not produced by mammals. The aspartic acid pathway is involved in the biosynthesis of a quarter of all amino acids required for protein synthesis and produces several critical metabolic intermediates. In particular, methionine, threonine and isoleucine are synthesized as branches off the aspartic acid biosynthesis pathway. The aspartic acid pathway is highly conserved in bacteria, fungi and plants but is not found in mammals, making key enzymes of this pathway attractive targets for drug discovery (Nazi et al., 2007).

Several molecules that affect microbial growth and infection by interfering with the aspartic acid pathway have been reported in the past few years. For example, the amino acid ana$\log (\mathrm{S})$-2-amino-5-hydroxnuc-4-oxopentanoic acid validates the idea of consideration of this pathway as a target. This compound inhibits homoserine dehydrogenase of the aspartic acid biosynthetic pathway. This compound hinders C. albicans infections in mice with no toxic effect to the host (Yamaki et al., 1990). Another example, azoxybacillin, produced by Bacillus cereus, is effective against the biosynthesis of sulphur containing amino acids. Rhizocticin A, from Bacillus subtilis, disrupts threonine biosynthesis (Yamaguchi et al., 1988). In the first step of the aspartic acid pathway, aspartate kinase phosphorylates aspartate to give aspartyl-phosphate. Aspartyl-phosphate is reduced in an NADPH-dependant manner to give aspartate 4-semialdehyde by the enzyme aspartate semialdehyde dehydrogenase. This reaction involves a nucleophilic attack followed by a hydride transfer from NADPH resulting in the formation of aspartate 4-semialdehyde. At this stage in plants and bacteria the pathway branches to synthesize either lysine or methionine, threonine, and isoleucine through homoserine, which is the only possible intermediate for fungi. In yeast, homoserine biosynthesis is achieved through the NADPH/ $\mathrm{NADH}$-dependent reduction of aspartate 4-semialdehyde by the action of homoserine dehydrogenase. The final common precursor of this pathway is homoserine, before the pathway branches in two different directions, the first to produce methionine and the second to produce threonine or isoleucine. In the methionine biosynthetic branch of the Asp pathway, the hydroxyl group of homoserine is activated by acylation. Two crucial acylating enzymes that convert homoserine to methionine are homoserine transacetylase and homoserine transsuccinylase. Both enzymes activate the hydroxyl group of homoserine for displacement of the corresponding acid by the thiol of cysteine to form cystathionine by the pyridoxal phosphate dependent enzyme cystathionine synthase.

\section{Homoserine transacetylase as a target for new antifun- gal drug development}

Fungal homoserine transacetylases are approximately 450 amino acids long and belong to the $\alpha / \beta$ hydrolase fold superfamily of enzymes that includes various esterases, lipases, and peptidases. This family is characterized by an eight-stranded sheet connected by helices with an active site catalytic triad plug. The nucleophilic residues in the catalytic triad can be serine, cysteine or glutamic acid (Born, Franklin, \& Blanchard, 

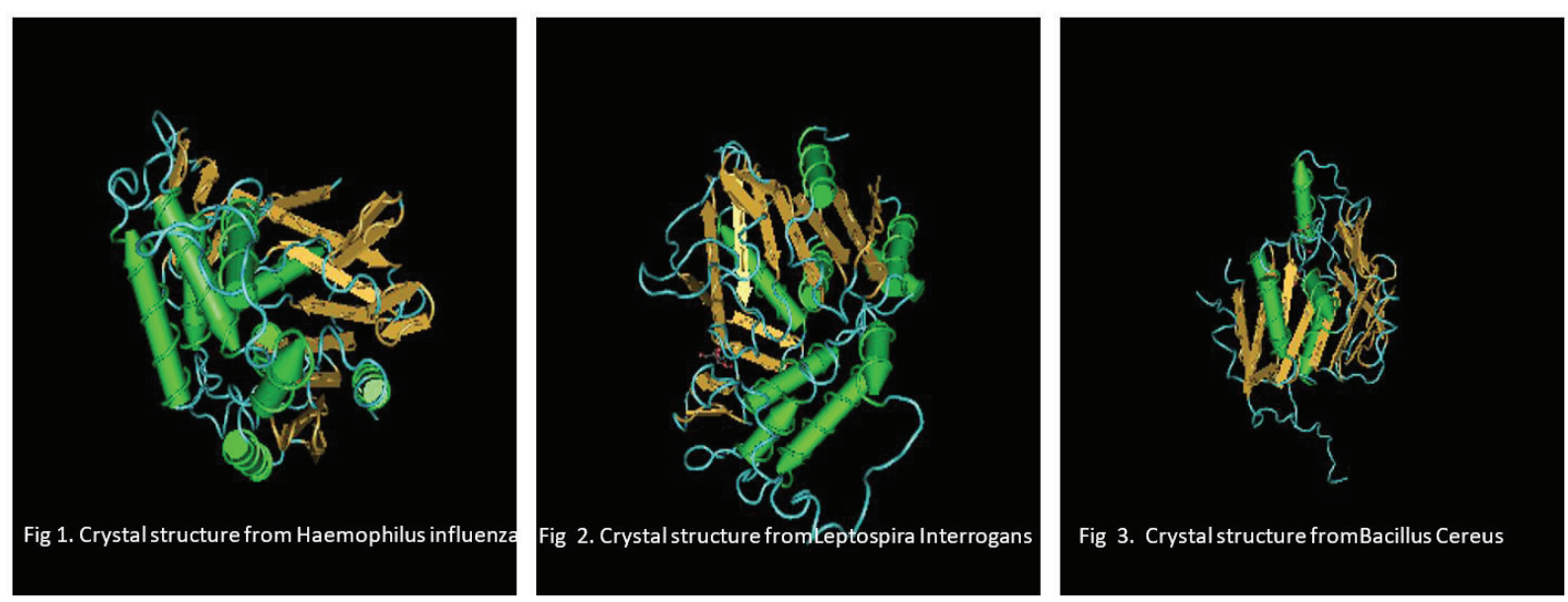

Figure 1-3. Clear differ 7 ences exist between structurally characterized examples of homoserine transacetylase.

2000; Ollis et al., 1992). Homoserine transacetylase catalyses the first committed step in the synthesis of methionine from aspartic acid. The Eenzyme catalyses the transfer of the acetyl group from acetyl coenzyme A to the hydroxyl group of homoserine. Early efforts were focused on isolation and purification of the enzyme. Homoserine transacetylase was initially purified from Bacillus polymyxa. Early kinetic studies revealed that the enzyme catalyses the transfer of the acetyl group through a ping-pong mechanism in which the acyl group of acetyl CoA is first transferred to an active site residue before subsequent transfer to the hydroxyl moiety of homoserine (Wyman \& Paulus, 1975). A ping-pong reaction is where in a two-substrate, two-product system an enzyme reacts with one substrate to form a product and a modified enzyme, the latter then reacts with a second substrate to form a second product and regenerates the original enzyme (Cleland, 1977). Born et al. showed that homoserine transacetylase from Haemophilus influenzae also undergoes a reaction with a pingpong kinetic mechanism; an initial acyl-enzyme intermediate is formed with acetyl-coA then the acetate is transferred to the hydroxyl group of homoserine (Born, et al., 2000). Homoserine transacetylase from the fission yeast Schizosaccharomyces pombe follows a covalent catalytic strategy to acetylate homoserine through an active site serine residue. Steadystate kinetic parameters provide evidence that enzyme from S. pombe also follows a ping-pong mechanism. Using site directed mutagenesis, site-specific mutants of the enzyme were constructed. Steady state kinetics study of the mutants demonstrated that Ser163, Asp403, and His432 are the likely active-site residues of a catalytic triad (Nazi, \& Wright, 2005). Crystal structures of prokaryotic forms of enzyme have been solved (Zager, 2000). Figures 1, 2 and 3 are generated using the Protein Structure Data Base. The overall detailed topology of these three proteins obviously differs from each other.

The details of the active site and how it might bind a drug are likely significantly different. In order to exploit the potential of homoserine transacetylase as an antifungal target, the structural basis of catalytic activation of homoserine transacetylase needs to be determined from C. albicans or from a phylogenetically closely related human pathogenic fungi.

\section{CONCLUSIONS}

The first step for drug discovery is target identification. A potent drug should have low host toxicity, ideally should not be found in host cells, and should be essential for the life of the pathogen while presenting the desired activity for inactivating targets. The conserved subset fungal amino acid biosynthetic enzymes which are absent in human presents an underexploited realm as antifungal drug targets. Particularly, the biosynthesis of methionine has been suggested to be susceptible to small molecule inhibition in fungi. Methionine is synthesized in bacteria, fungi, and plants. The first committed step in methionine biosynthesis is identified as the acetylation of homoserine by the enzyme homoserine transacetylase, a promising drug-susceptible target for new antifungal drug development.

Peer-review: Externally peer-reviewed

Author Contributions: Conception/Design of Study- E.S.; Data Acquisition- E.S.; Data Analysis/Interpretation- E.S.; Drafting Manuscript- E.S.; Critical Revision of Manuscript- E.S.; Final Approval and Accountability- E.S.

Conflict of Interest: The authors have no conflict of interest to declare.

Financial Disclosure: Authors declared no financial support.

\section{REFERENCES}

Ashman, R. B., Farah, C. S., Wanasaengsakul, S., Hu, Y., Pang, G., \& Clancy, R. L. (2004). Innate versus adaptive immunity in Candida albicans infection. Immunology and Cell Biology, 82(2), 196-204. https://doi.org/10.1046/j.0818-9641.2004.01217.x

Born, T. L., Franklin, M., \& Blanchard, J. S. (2000). Enzyme-catalyzed acylation of homoserine: mechanistic characterization of the Haemophilus influenzae met2-encoded homoserine transacetylase. Biochemistry, 39(29), 8556-8564. https://doi.org/10.1021/ bi000462p

Chang, Y. L., Yu, S. J., Heitman, J., Wellington, M., \& Chen, Y. L. (2017). New facets of antifungal therapy. Virulence, 8(2), 222-236. https://doi.org/10.1080/21505594.2016.1257457

- Cleland W. W. (1977). Determining the chemical mechanisms of enzyme-catalyzed reactions by kinetic studies. Advances in Enzymology and Related Areas of Molecular Biology, 45, 273-387. https://doi.org/10.1002/9780470122907.ch4 
- Hollomon D. W. (1993). Resistance to azole fungicides in the field. Biochemical Society Transactions, 21(4), 1047-1051. https:// doi.org/10.1042/bst0211047

- Low, C. Y., \& Rotstein, C. (2011). Emerging fungal infections in immunocompromised patients. F1000 Medicine Reports, 3, 14. https://doi.org/10.3410/M3-14

- Mazu, T. K., Bricker, B. A., Flores-Rozas, H., \& Ablordeppey, S. Y. (2016). The Mechanistic Targets of Antifungal Agents: An Overview. Mini Reviews in Medicinal Chemistry, 16(7), 555-578. https:// doi.org/10.2174/1389557516666160118112103

- Nazi, I., \& Wright, G. D. (2005). Catalytic mechanism of fungal homoserine transacetylase. Biochemistry, 44(41), 13560-13566. https://doi.org/10.1021/bi0514764

- Nazi, I., Scott, A., Sham, A., Rossi, L., Williamson, P. R., Kronstad, J. W., \&Wright, G. D. (2007). Role of homoserine transacetylase as a new target for antifungal agents. Antimicrobial agents and chemotherapy, 51(5), 1731-1736. https://doi.org/10.1128/AAC.01400-06

- Ollis, D. L., Cheah, E., Cygler, M., Dijkstra, B., Frolow, F., Franken, S. M., Schrag, J. (1992). The alpha/beta hydrolase fold. Protein Engineering, 5(3), 197-211. https://doi.org/10.1093/protein/5.3.197

- $\quad$ Omura S., \& Tanaka H. (1984). Production, structure, and antifungal activity of polyene macrolides, , In S. Omura (Ed.), Macrolide Antibiotics: Chemistry, Biology, and Practice (pp. 351-405). New York, USA: Academic Press.

- $\quad$ Panackal, A. A., Gribskov, J. L., Staab, J. F., Kirby, K. A., Rinaldi, M., \& Marr, K. A. (2006). Clinical significance of azole antifungal drug crossresistance in Candida glabrata. Journal of Clinical Microbiology, 44(5), 1740-1743. https://doi.org/10.1128/JCM.44.5.1740-1743.2006

- $\quad$ Pfaller, M. A., \& Diekema, D. J. (2007). Epidemiology of invasive candidiasis: a persistent public health problem. Clinical Microbiology Reviews, 20(1), 133-163. https://doi.org/10.1128/CMR.00029-06

- Vandeputte, P., Larcher, G., Bergès, T., Renier, G., Chabasse, D., \& Bouchara, J. P. (2005). Mechanisms of azole resistance in a clinical isolate of Candida tropicalis. Antimicrobial Agents and Chemotherapy, 49(11), 4608-4615. https://doi.org/10.1128/AAC.49.11.46084615.2005
Warrilow, A. G., Nishimoto, A. T., Parker, J. E., Price, C. L., Flowers, S. A., Kelly, D. E., Kelly, S. L. (2019). The Evolution of Azole Resistance in Candida albicans Sterol 14a-Demethylase (CYP51) through Incremental Amino Acid Substitutions. Antimicrobial Agents and Chemotherapy, 63(5), e02586-18. https://doi.org/10.1128/ AAC.02586-18

Whaley, S. G., Berkow, E. L., Rybak, J. M., Nishimoto, A. T., Barker, K. S., \& Rogers, P. D. (2016). Azole Antifungal Resistance in Candida albicans and Emerging Non-albicans Candida Species. Frontiers in Microbiology, 7, 2173. https://doi.org/10.3389/fmicb.2016.02173

Wyman, A., \& Paulus, H. (1975). Purification and properties of homoserine transacetylase from Bacillus polymyxa. The Journal of Biological Chemistry, 250(10), 3897-3903.

- Yamaguchi, H., Uchida, K., Hiratani, T., Nagate, T., Watanabe, N., \& Omura, S. (1988). RI-331, a new antifungal antibiotic. Annals of the New York Academy of Sciences, 544, 188-190. https://doi. org/10.1111/j.1749-6632.1988.tb40403.x

- Yamaki, H., Yamaguchi, M., Imamura, H., Suzuki, H., Nishimura, T., Saito, H., \& Yamaguchi, H. (1990). The mechanism of antifungal action of (S)-2-amino-4-oxo-5-hydroxypentanoic acid, Rl-331: the inhibition of homoserine dehydrogenase in Saccharomyces cerevisiae. Biochemical and Biophysical Research Communications, 168(2), 837-843. https://doi.org/10.1016/0006291x(90)92397-i

Zager R. A. (2000). Polyene antibiotics: relative degrees of in vitro cytotoxicity and potential effects on tubule phospholipid and ceramide content. American Journal of Kidney Diseases : the official journal of the National Kidney Foundation, 36(2), 238-249. https:// doi.org/10.1053/ajkd.2000.8967 